Micaela Ödman · Bertrand Jaques

Kathleen Meagher-Villemure $\cdot$ Philippe Pasche

\title{
Myxoid fibroblastic tumor of the maxillary sinus in a 21-month-old child: an unusual diagnosis
}

Received: 4 November 2005/ Accepted: 8 February 2006/Published online: 5 May 2006

(C) Springer-Verlag 2006

\begin{abstract}
Myxoid tumors of the soft tissue are a heterogeneous group of lesions with significant differences in behavior, from being totally harmless to malignant tumors with metastasizing potential. We describe the unusual case of a 21-month-old boy who developed a paranasal low-grade myxoid neoplasm with recurrent potential. The histological findings showed a proliferation of spindle cells within a vascular and myxoid background. The immunohistochemistry had the features of myofibroblasts and fibroblasts with strong Vimentin and light Smooth Muscle Actin reactivity. Close collaboration between clinician and pathologist is necessary to deal with these unusual lesions to predict their local aggressiveness and long-term behavior. Most of these cellular myxomas have been described in adults, involving primarily the limbs, but this case shows that any localization is possible.
\end{abstract}

Keywords Paranasal sinus neoplasms · Connective and soft tissue neoplasms $\cdot$ Vimentin

\section{Clinical summary}

A healthy 21-month-old boy fell on his nose, resulting in light bruising. In the following days, the parents noticed a discrete residual asymmetry. Two weeks later, the boy

M. Ödman $(\bowtie) \cdot$ P. Pasche

Department of Otorhinolaryngology and Cervico-Facial Surgery, Centre Hospitalier Universitaire Vaudois, Lausanne, Switzerland E-mail: Micaela.Oedman@chuv.ch

Tel.: + 41-21-3142688

Fax: + 41-21-3142646

B. Jaques

Department of Maxillofacial Surgery,

Centre Hospitalier Universitaire Vaudois,

Lausanne, Switzerland

K. Meagher-Villemure

University Institute of Pathology,

Centre Hospitalier Universitaire Vaudois,

Lausanne, Switzerland was seen by an otorhinolaryngologist. The child had a $2-\mathrm{cm}$ large, firm and painless right paranasal bump, without skin discoloration. The bump was mobile to the skin and fixed to the deep plane. Rhinoscopy was normal. A plain X-ray showed homogenous density of the lesion, interpreted as an old hematoma. The boy was re-examined 1 month later. The examination showed that the bump had slightly increased in size; otherwise the clinical examination was essentially normal. A CT scan was performed, which revealed an expansive mass in communication with the ethmoid sinus and the nose, compressing the lacrymal apparatus. To clarify the diagnosis, an MRI was done and showed a large hypodense mass in the right maxillary sinus extending to both ethmoids and subcutaneous tissue, enhancing with contrast (Figs. 1, 2). A biopsy of this mass was performed under general anesthesia, followed 1 month later by a macroscopic total excision by a combined endonasal, transconjunctival and vestibular approach. The orbital floor was partially removed, along with the anterior ethmoidal cells and the superior naso-sinusal wall. The resection margins were positive, and the follow-up consisted of rhinoscopy and an MRI every 6 months. One year after the first extensive surgical excision, a newly grown myxoid tissue was noticed in the anterior ethmoid cells and maxillary sinus and was excised by an endonasal approach, showing the same histological features. Two years after the last excision, clinical examination and MRI performed every 6 months revealed no recurrence.

\section{Pathology}

All material obtained from the first biopsy to the extensive surgical resection and the recurrence 1 year later showed the same histology. It consisted of a moderate cellular proliferation of thin spindle cells with occasional mucoid microcysts and a vascular and myxoid background. The spindle cells were composed of oval or round nuclei and scanty eosinophilic cytoplasm. 


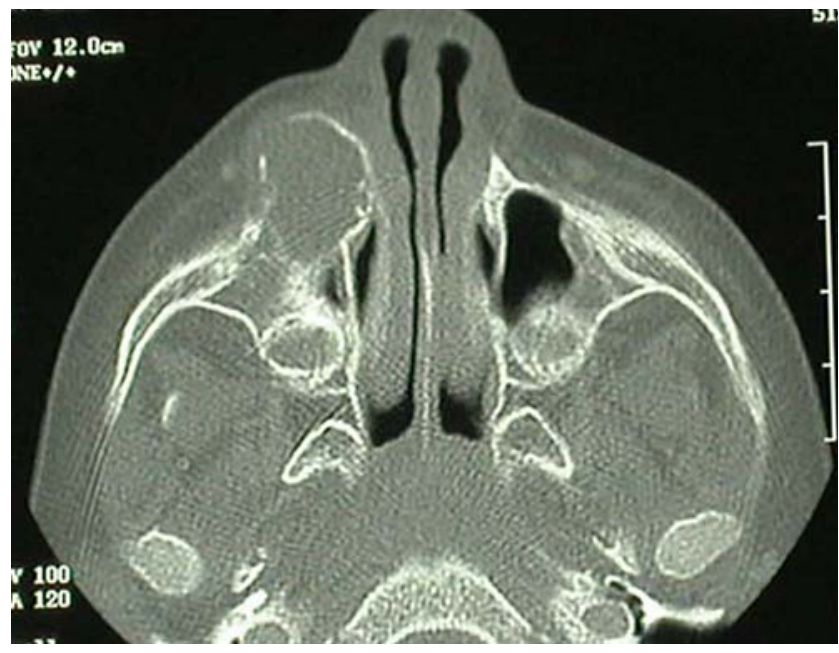

Fig. 1 Facial CT-scan: axial plane
These cells were dispersed in a prominent myxoid matrix with focal collagen fibers (Fig. 3). Mitosis were present in a distribution of one per 5-10 high power fields. There was no atypical mitosis or area of necrosis. In some areas, there was a subtle whorled pattern. On immunohistochemistry, the tumor cells showed strong diffuse reactivity for Vimentin, weak diffuse positivity for Muscle Specific Actin and weak focal reactivity for Smooth Muscle Actin. The tumor cells were negative for Desmin, CD34, Myogenin, Glial fibrillary acidic Protein and S-100 protein. The ultrastructural study was not significantly contributory as it revealed non-cohesive tumor cells with features of fibroblasts consisting of significant dilated rough endoplasmic reticulum, few mitochondria and microfilaments without specific organization. Between the cells, the matrix was loose and contained only small electron dense debris. Based upon these findings, the tumor was considered as a
Fig. 2 Facial MRI: sagittal and axial planes
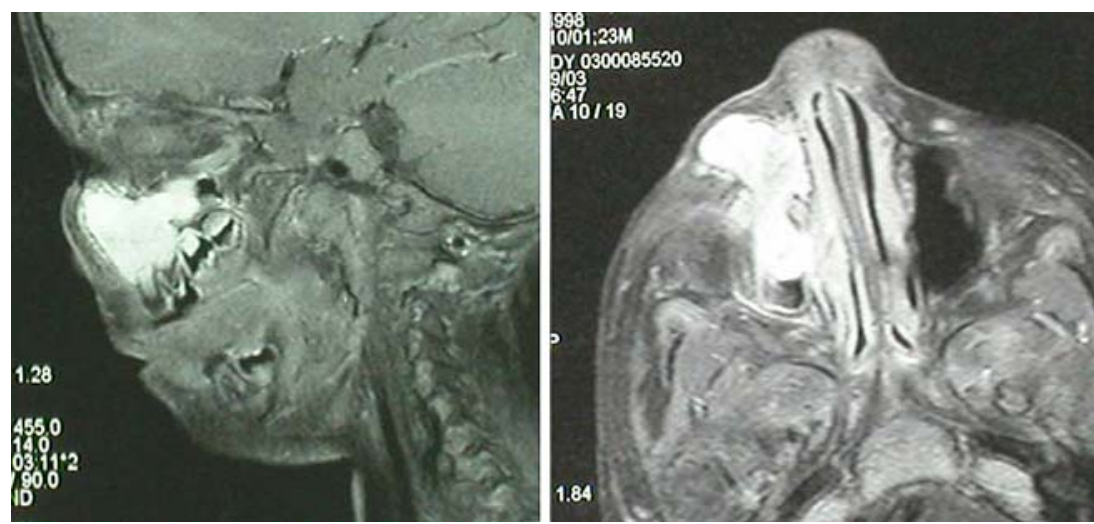

Fig. 3 a Moderately cellular proliferation of spindle cells, small vessels in a myxoid background, $\mathrm{HE} \times 100$. b Proliferation of spindle cells with thin eosinophilic cytoplasm and a rare mitotic figure, $\mathrm{HE} \times 450$. c Masson's trichrome stain revealing fibroblasts in blue and myofibroblasts in red, $\times 450$. d Immunohistochemistry for Vimentin strong positive reaction, $\times 450$

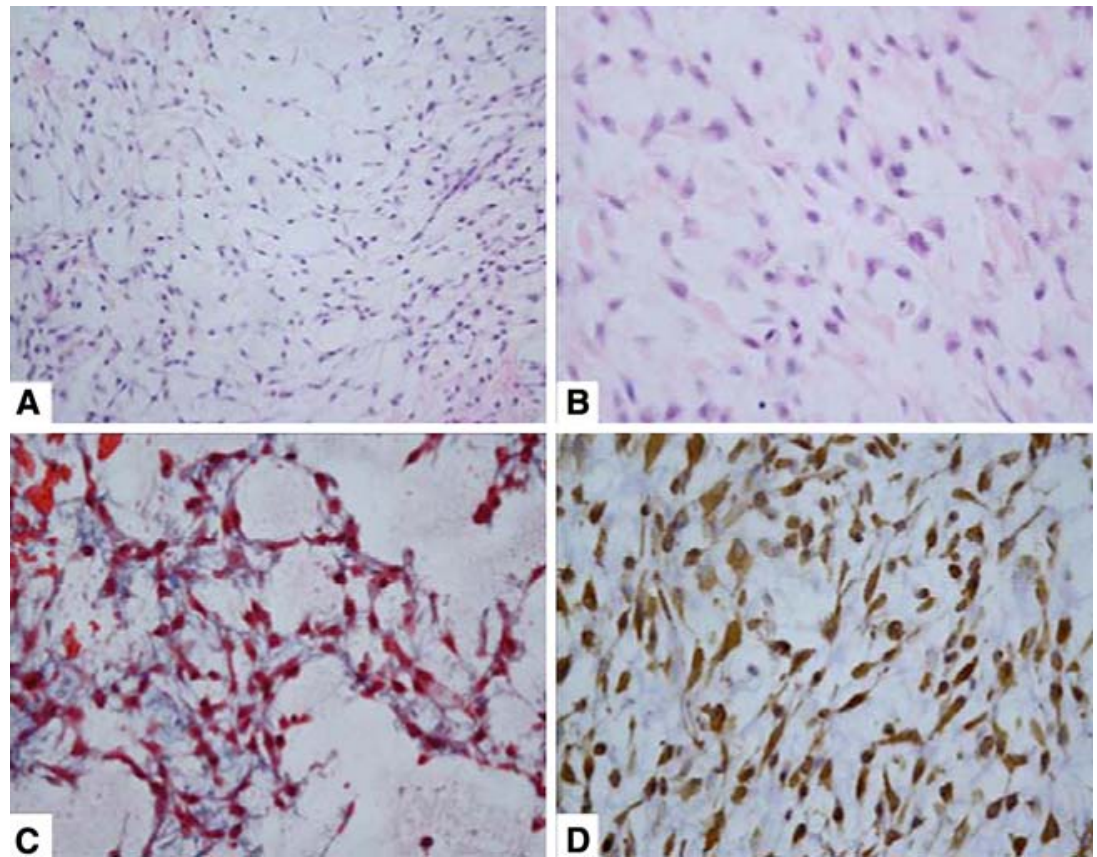


low-grade myxoid neoplasm with a fibroblastic-myofibroblastic phenotype.

\section{Discussion}

A very extensive and detailed review of myxoid tumors was published in 1999 [1]. These lesions are described as a heterogeneous group of tumors with an abundant extracellular myxoid matrix. They include a large range of different biological behaviors, from entirely harmless lesions to malignant tumors. The low-grade myxoid neoplasm with recurrent potential (or cellular myxoma) is used to denote lesions with features intermediate between intramuscular myxoma and low-grade myxofibrosarcoma. It is described as a slowly growing subcutaneous, subfacial or intramuscular mass most often located in the limbs, more rarely found on the trunk, the perineum or the retroperitoneal region and almost exclusively seen in adults. These tumors may recur locally $(5-10 \%)$ but never reach a high histological grade nor do they metastasize. Histologically, no nuclear atypia or cytological pleomorphism is seen, and multinucleate cells are generally absent. On immunohistochemistry, the tumor cells are reactive with antibodies such as Vimentin and Smooth Muscle Actin and occasionally with CD34; they are negative for S-100 Protein and Desmin. A review article published in 2000 provides very detailed information about myxoid lesions [2]. Thirty-eight cases of cellular myxoma were described in 2001 [3], none of which resembles our case. They all occurred in adults aged 25-83 years, and most of them involved the limbs. One single lesion arose on the cheek. In reviewing the immunohistochemistry reactions, CD34 positivity was identified in $57 \%$ of the cases, Smooth Muscle Actin reaction was positive in $10 \%$ and Desmin and S100 protein were always negative. A 1997 study reported 19 mixed tumors of soft tissue and described the variety of localization and of patients suffering from these lesions [4]. According to the literature, the localization of these tumors very seldom involves the face. A case of leiomyoma of the nasal septum was described in 1996, with a review of the literature reporting only 19 cases between 1966 and 1996 [5]. An additional case was reported in 2002 [6]. To our knowledge, no case similar to the one we are reporting has been described. Despite the aggressiveness of the tumor, it is considered as benign, which explains our conservative attitude, considering its close proximity to the eye. Further endonasal excision or biopsy may be necessary in the future based upon clinical and radiological findings during the follow-up.

\section{Conclusion}

It appears that cellular myxomas, or low-grade myxoid neoplasms with recurrent potential are a heterogeneous group of lesions, occurring mostly in the limbs, but also involving the head-and-neck region, and arising at any age. Diagnosis is provided by histology and immunohistochemistry, which allows differentiation of the types of myxoid tumors. This is of major importance for treatment and follow-up because of the different biological behavior of myxoid tumors.

Acknowledgements We would like to thank Professor Cheryl M.Coffin for reviewing the material from this case and providing her opinion on the management of this type of tumor based upon her experience.

\section{References}

1. van Roggen G, Fletcher H, Fletcher M (1999) Myxoid tumours of soft tissue. Histopathology 35(4):291-312

2. Allen PW (2000) Myxoma is not a single entity: a review of the concept of Myxoma. Ann Diagn Pathol 4:99-123

3. van Roggen JFG, McMenamin ME, Fletcher CDM (2001) Cellular myxoma of soft tissue: a clinicopathological study of 38 cases confirming indolent clinical behaviour. Histopathology 39(3):287-297

4. Kilpatrick SE, Hitchcock MG, Kraus MD, Calonje E, Fletcher CDM (1997) Mixed tumours and myoepitheliomas of soft tissue: a clinicopathological study of 19 cases with a unifying concept. Am J Surg Pathol 21:13-22

5. Llorente JL, Suarez C, Seco M, Garcia A (1996) Leiomyoma of the nasal septum: report of a case and review of the literature. J Laryngol Otol 110:65-68

6. Vincenzi A, Rossi G, Monzani D, Longo L, Rivasi F (2002) Atypical (bizarre) leiomyoma of the nasal cavity with prominent myxoid change. J Clin Pathol 55:872-875 